\title{
SPECTRAL AND ASYMPTOTIC PROPERTIES OF DOMINATED OPERATORS
}

\author{
FRANK RÄBIGER and MANFRED P. H. WOLFF
}

(Received 1 November 1995; revised 26 July 1996)

Communicated by P. G. Dodds

\begin{abstract}
We investigate the relationship between the peripheral spectrum of a positive operator $T$ on a Banach lattice $E$ and the peripheral spectrum of the operators $S$ dominated by $T$, that is, $|S x| \leq T|x|$ for all $x \in E$. This can be applied to obtain inheritance results for asymptotic properties of dominated operators.

1991 Mathematics subject classification (Amer. Math. Soc.): primary 47B65, 47A10, 47A35, 47A53.

Keywords and phrases: Banach lattice, dominated operators, peripheral spectrum, essential spectrum, almost periodicity, uniform ergodicity.
\end{abstract}

\section{Introduction}

The investigation of operators on Banach lattices leads to the natural question which properties of a positive operator $T$ on a Banach lattice $E$ are inherited by the operators $S$ dominated by $T$, that is, $|S x| \leq T|x|$ for all $x \in E$. For certain properties one has to impose the additional assumption, that the operator $S$ is also positive.

There are numerous results on inheritance of operator properties such as compactness, weak compactness, or being a kernel or a Dunford-Pettis operator (see, for example, $[1,4,7,9,13,14,20,27]$; see also $[2,19,22,28]$ for a comprehensive survey and further developments). Only recently the inheritance of spectral and asymptotic properties of an operator has been investigated (see, for example, $[3,5,17,18,21$, 23-25]).

In the present paper we are mainly interested in properties of the peripheral spectrum of a dominated operator. We always assume that the dominating operator $T$ satisfies a certain growth condition $(G)$. Then for positive operators $S$ dominated by $T$ one has

This paper is part of a research project supported by the Deutsche Forschungsgemeinschaft DFG

(C) 1997 Australian Mathematical Society 0263-6115/97\$A2.00+0.00 
$\sigma(S) \cap r(T) \Gamma \subseteq \sigma(T) \cap r(T) \Gamma$, that is, either the spectral radii satisfy $r(S)<r(T)$ or the peripheral spectrum $\sigma(S) \cap r(S) \Gamma$ of $S$ is contained in the peripheral spectrum $\sigma(T) \cap r(T) \Gamma$ of $T$ (see Theorem 1.4). If $T$ satisfies an ergodicity condition and/or the Banach lattice $E$ has order continuous norm or is a $K B$-space, one obtains the corresponding inclusion $P \sigma(S) \cap r(T) \Gamma \subseteq P \sigma(T) \cap r(T) \Gamma$ for the point spectrum (see Theorem 2.2, Corollary 2.4 and Theorem 2.6). If $r(T)$ is a Riesz point of $T$ and $S$ a (not necessarily positive) operator dominated by $T$, then $r(S)<r(T)$ or the peripheral spectrum of $S$ contains only Riesz points (see Theorem 3.1). This generalizes a result of Caselles [5, Theorem 4.1], where $S$ is assumed to be positive. Finally we apply the above results and investigate inheritance of asymptotic properties such as uniform convergence of $S^{n+1}-S^{n}$ to 0 (see Theorem 4.1), almost periodicity and strong convergence of the powers $S^{n}$ (see Theorem 4.2 and Corollary 4.3), and uniform ergodicity of $S$ (see Theorem 4.5). In particular we generalize a result of Caselles [5, Corollary 4.6] and extend results of Räbiger [24, 25].

Our notation is standard and follows mainly the books of Meyer-Nieberg [19] and Schaefer [26]. Unexplained terminology can be found there. We briefly recall some frequently used notions. By $\Gamma:=\{\lambda \in \mathbb{C}:|\lambda|=1\}$ we denote the unit circle. Throughout the whole paper we consider spaces over $\mathbb{C}$. If $E$ is a Banach space, then $\mathscr{L}(E)$ is the space of all bounded linear operators on $E$ and $E^{\prime}$ the (topological) dual of $E$. For $T \in \mathscr{L}(E)$ let $T^{\prime} \in \mathscr{L}\left(E^{\prime}\right)$ be the adjoint of $T$. Moreover, $\sigma(T)$ denotes the spectrum, $r(T):=\sup \{|\lambda|: \lambda \in \sigma(T)\}$ the spectral radius, $P \sigma(T):=\{\lambda \in \sigma(T): \lambda$ is an eigenvalue of $T\}$ the point spectrum, and $\rho(T):=\mathbb{C} \backslash \sigma(T)$ the resolvent set of $T$. For $\lambda \in \rho(T)$ we set $R(\lambda, T):=(\lambda I-T)^{-1}$.

Now let $E$ be a (complex) Banach lattice with modulus $\mid$. $\mid$. Then $E_{+}:=\{x \in E$ : $x=|x|\}$ is the set of all positive elements in $E$. The dual space $E^{\prime}$ is again a Banach lattice and $x^{\prime} \in E^{\prime}$ is positive if and only if $\left\langle x^{\prime}, x\right\rangle \geq 0$ for all $x \in E_{+}$. For operators $S, T \in \mathscr{L}(E)$ we write $S \leq T$ if $(T-S) E_{+} \subseteq E_{+}$, and $T$ is called positive if $0 \leq T$.

\section{The peripheral spectrum of dominated positive operators}

In this section we show that for operators $0 \leq S \leq T$ on a Banach lattice $E$ one always has $\sigma(S) \cap r(T) \Gamma \subseteq \sigma(T) \cap r(T) \Gamma$ provided that $T$ satisfies a certain growth condition $(G)$.

At first we recall some well-known facts and fix some notations. Let $T \in \mathscr{L}(E)$ be a bounded linear operator on a Banach space $E$. If $G$ is a closed linear subspace of $E$ such that $T G \subseteq G$ we denote by $T_{1}=T_{\mid G}$ the restriction of $T$ to $G$ and by $T_{/}=T_{/ G}$ the induced operator on the quotient space $E / G$ given by $T_{/}(x+G):=T x+G, x \in E$. The following lemma is well-known (see [26, V, Exercise 5]). 
LEMMA 1.1. Under the conditions above one has

(i) $\left(\sigma\left(T_{\mid}\right) \cup \sigma\left(T_{l}\right)\right) \cap r(T) \Gamma \subseteq \sigma(T) \cap r(T) \Gamma$.

(ii) $\max \left(r\left(T_{\mid}\right), r\left(T_{\rangle}\right)\right)=r(T)$.

(iii) $\lambda \in r(T) \Gamma$ is a pole of the resolvent $R(., T)$ (of order $k$ ) if and only if $\lambda$ is a pole of $R\left(., T_{1}\right)$ and $R\left(., T_{l}\right)$ (of order $k_{1}$ and $k_{l}$, respectively), and then $\sup \left(k_{1}, k_{l}\right) \leq$ $k \leq k_{1}+k_{1}$. Moreover, if $P$ is the residuum of $R(., T)$ at $\lambda$, then $P G \subseteq G$ and $P_{\mid}$and $P_{/}$is the residuum of $R\left(., T_{\mid}\right)$and $R\left(., T_{/}\right)$at $\lambda$, respectively.

In the sequel we make use of the following construction. For details we refer to [26, V.1]. If $E$ is a Banach space let $l_{\infty}(E)$ be the space of bounded $E$-valued sequences endowed with the sup-norm. For a free ultrafilter $\mathscr{U}$ on $\mathbb{N}$ we consider the closed subspace $c_{\mathscr{U}}(E):=\left\{\left(x_{n}\right) \in l_{\infty}(E): \lim _{\mathscr{U}}\left\|x_{n}\right\|=0\right\}$. The quotient space $E_{\mathscr{U}}:=l_{\infty}(E) / c_{\mathscr{U}}(E)$ is called ultrapower or $\mathscr{U}$-power of $E$. For $\left(x_{n}\right)+c_{\mathscr{U}}(E) \in E_{\mathscr{U}}$ we also write $\widehat{\left(x_{n}\right)}$. The mapping $x \mapsto(x, x, \ldots)$ is an isometric embedding of $E$ into $E_{\mathscr{U}}$ and thus $E$ can be considered a closed subspace of $E_{\mathscr{U}}$. Every operator $T \in \mathscr{L}(E)$ induces an operator $T_{\mathscr{U}} \in \mathscr{L}\left(E_{\mathscr{U}}\right)$ by means of $T_{\mathscr{U}} \widehat{\left(x_{n}\right)}:=\widehat{\left(T x_{n}\right)}$. Its restriction to $E$ satisfies $T_{\mathscr{U} \mid E}=T$. Moreover, the following holds (see [26, V.1]).

LEMMA 1.2. (i) $\left\|T_{\mathscr{Z}}\right\|=\|T\|$.

(ii) $\sigma\left(T_{\mathscr{U}}\right)=\sigma(T)$.

(iii) $\sigma\left(T_{\mathscr{U}}\right) \cap r(T) \Gamma \subseteq P \sigma\left(T_{\mathscr{U}}\right)$.

(vi) $R\left(\lambda, T_{\mathscr{U}}\right)=R(\lambda, T)_{\mathscr{U}}$ for all $\lambda \in \rho(T)$.

(v) $\lambda \in \sigma(T)$ is a pole of order $k$ of $R(., T)$ if and only if the same holds for $R\left(., T_{\mathscr{U}}\right)$.

If $E$ is a Banach lattice and $T \in \mathscr{L}(E)$ is a positive operator, then $E_{\mathscr{U}}$ is again a Banach lattice and $T_{\mathscr{U}} \in \mathscr{L}\left(E_{\mathscr{U}}\right)$ is positive. For $\widehat{\left(x_{n}\right)} \in E_{\mathscr{U}}$ one has $\left|\widehat{\left(x_{n}\right)}\right|=\widehat{\left(\left|x_{n}\right|\right)}$.

Now let $0 \leq S \leq T$ be operators on a Banach lattice $E$. In the following lemma we present a condition under which an eigenvalue of $S$ is also an eigenvalue of $T$.

LEMMA 1.3. Let $E$ be a Banach lattice and let $S, T \in \mathscr{L}(E)$ be such that $0 \leq S \leq$ $T$. Suppose there is $\alpha \in \Gamma$ and $x \in E$ such that $S x=\alpha x$ and $T|x|=|x|$. Then $T x=\alpha x$.

PROOF. The assumptions imply $|x| \leq S|x| \leq T|x|=|x|$. Thus $0 \leq|(T-S) x| \leq$ $(T-S)|x|=|x|-S|x| \leq 0$, and hence $T x=S x=\alpha x$.

REMARK. The conditions of the lemma are satisfied if $0 \leq S \leq T, S x=\alpha x$ for $\alpha \in \Gamma$ and $x \in E$, and there is a strictly positive linear form $x^{\prime} \in E_{+}^{\prime}$ such that $T^{\prime} x^{\prime} \leq x^{\prime}$. (Recall that $x^{\prime} \in E_{+}^{\prime}$ is strictly positive if $\left\langle x^{\prime}, y\right\rangle>0$ for all $y \in E_{+} \backslash\{0\}$.) In fact, from $0 \leq T|x|-|x|$ and $0 \leq\left\langle T|x|-|x|, x^{\prime}\right\rangle=\left\langle|x|,\left(T^{\prime}-I\right) x^{\prime}\right\rangle \leq 0$ we obtain $T|x|=|x|$ by the strict positivity of $x^{\prime}$. 
Now we come to the main result of this section. Recall that an operator $T$ on a Banach space $E$ satisfies the growth condition $(G)$ if $\lim \sup _{\lambda \downarrow r(T)}\|(\lambda-r(T)) R(\lambda, T)\|$ $<\infty$. Lemma 1.2 implies that then $T_{\mathscr{U}} \in \mathscr{L}\left(E_{\mathscr{U}}\right)$ has also property $(G)$ for every ultrapower $E_{\mathscr{U}}$ of $E$. Clearly every operator with uniformly bounded powers and spectral radius 1 satisfies $(G)$. Moreover a positive operator $T$ on a Banach lattice $E$ with $r(T)=1$ has property $(G)$ if and only if the Cesaro means $T_{n}:=$ $n^{-1} \sum_{k=0}^{n-1} T^{k}, n \in \mathbb{N}$, are uniformly bounded (see $[10,1.5,1.7]$ ).

THEOREM 1.4. Let $E$ be a Banach lattice and let $S, T \in \mathscr{L}(E)$ such that $0 \leq S \leq T$ and $T$ satisfies $(G)$. Then $\sigma(S) \cap r(T) \Gamma \subseteq \sigma(T) \cap r(T) \Gamma$.

ProOF. The assumptions imply $0 \leq r(S) \leq r(T)$. If $r(T)=0$ there is nothing to prove. Otherwise we may assume $r(T)=1$ and, by passing to an ultrapower, $\sigma(S) \cap \Gamma \subseteq P \sigma(S)$. Let $\alpha \in \sigma(S) \cap \Gamma$ and choose $0 \neq x \in E$ such that $S x=\alpha x$. Then $|x| \leq S|x| \leq T|x|$. For $y \in E$ let $p(y):=\lim \sup _{\lambda \downarrow 1}(\lambda-1)\|R(\lambda, T)|y|\|$ (see also [19, proof of 4.1.11]). Since $T$ satisfies $(G)$ the mapping $p$ is a continuous lattice seminorm. Then $J:=\operatorname{ker} p$ is a closed ideal in $E$. From $p(T y) \leq\|T\| p(y)$ we obtain $T J \subseteq J$ and $S J \subseteq J$. Let $S$, and $T$, be the operators on $E / J$ induced by $S$ and $T$, respectively. From $|x| \leq T|x|$ it follows that $p(x) \geq\|x\|>0$, and hence $\tilde{x}:=x+J \neq 0$. Clearly $S_{/} \tilde{x}=\alpha \tilde{x}$. Moreover, since $T$ satisfies $(G)$,

$$
\begin{aligned}
p(T|x|-|x|) & =\limsup _{\lambda \downarrow 1}(\lambda-1)\|R(\lambda, T)(T-\lambda+\lambda-1)|x|\| \\
& =\limsup _{\lambda \downarrow 1}(\lambda-1)^{2}\|R(\lambda, T)|x|\|=0 .
\end{aligned}
$$

Thus $T|x|-|x| \in J$; that is, $T_{/}|\tilde{x}|=|\tilde{x}|$. Now Lemma 1.3 implies $T_{/} \tilde{x}=\alpha \tilde{x}$. Hence $\alpha \in \sigma(T)$ by Lemma 1.1.

As in the proof of [26, V.4.9], one can extend Theorem 1.4 to operators $T$ which are $(G)$-solvable. Recall that a positive operator $T$ on a Banach lattice $E$ is $(G)$-solvable, if there exist finitely many closed $T$-invariant ideals $\{0\}=I_{1} \subseteq I_{2} \subseteq \cdots \subseteq I_{n}=E$ such that the operator $T_{k}$ induced on $I_{k} / I_{k-1}$ satisfies $(G)$ for all $2 \leq k \leq n$.

COROLLARY 1.5. Let $E$ be a Banach lattice and $S, T \in \mathscr{L}(E)$ operators such that $0 \leq S \leq T$. If $T$ is $(G)$-solvable, then $\sigma(S) \cap r(T) \Gamma \subseteq \sigma(T) \cap r(T) \Gamma$.

If $T$ is a positive operator and $r(T)$ is a pole of the resolvent map $\lambda \mapsto R(\lambda, T)$, then $T$ is $(G)$-solvable (see [26, p.326, Example 4]). In particular, this is the case if $r(T)$ is a Riesz point of $T$, that is, a pole of the resolvent map $R(., T)$ with finite dimensional residuum. 
COROLLARY 1.6. Let $E$ be a Banach lattice and $S, T \in \mathscr{L}(E)$ operators such that $0 \leq S \leq T$. If $r(T)$ is a Riesz point, then $\sigma(S) \cap r(T) \Gamma \subseteq \sigma(T) \cap r(T) \Gamma$.

REMARK. If $r(S)=r(T)$, then by a result of Caselles [5, Theorem 4.1] $r(S)$ is a Riesz point of $S$, and hence $\sigma(S) \cap r(S) \Gamma$ consists entirely of Riesz points (see [26, V.5.5]). In Theorem 3.1 we will show that this conclusion actually holds for any operator $S$ such that $|S x| \leq T|x|$ for all $x \in E$.

\section{The peripheral point spectrum of dominated positive operators}

In this section we give analogues of Theorem 1.4 for the point spectrum. At first we recall some well-known facts from ergodic theory and the theory of Banach lattices. The following proposition is a special case of a general ergodic theorem due to Eberlein [9, Theorem 3.1].

PROPOSITION 2.1. Let $T \in \mathscr{L}(E)$ be an operator on a Banach space $E$ and suppose that $r(T)=1$ and $T$ satisfies $(G)$. Then for $x \in E$ the following assertions are equivalent:

(i) $\lim _{\lambda \downarrow 1}(\lambda-1) R(\lambda, T) x$ exists in $E$.

(ii) $((\lambda-1) R(\lambda, T) x)_{\lambda>1}$ has a weak cluster point (as $\lambda \rightarrow 1$ ).

In this case $y:=\lim _{\lambda \downarrow 1}(\lambda-1) R(\lambda, T) x$ satisfies $T y=y$.

An operator $T \in \mathscr{L}(E)$ is called Abel ergodic if

$$
P_{T} x:=\lim _{\lambda \downarrow r(T)}(\lambda-r(T)) R(\lambda, T) x \text { exists for all } x \in E .
$$

From $(\lambda-r(T)) R(\lambda, T)=(a \lambda-\operatorname{ar}(T)) R(a \lambda, a T), \lambda \in \rho(T), a>0$, it follows that $T$ is Abel ergodic if and only if $a T$ is Abel ergodic for all $a>0$. If $T$ is Abel ergodic and $r(T)>0$, then $P_{T} \in \mathscr{L}(E)$ is a projection, $P_{T} E=\{x \in E: T x=r(T) x\}$, and $\operatorname{ker} P_{T}=\overline{(r(T)-T) E}$ (see [16, 2.1.9]). By the uniform boundedness principle, every Abel ergodic operator satisfies $(G)$.

For our next theorem we need a construction from the theory of Banach lattices (see [26, II.8, Example 1]). Let $E$ be a Banach lattice and $y^{\prime} \in E_{+}^{\prime}$. The mapping $p: E \rightarrow \mathbb{R}_{+} ; x \mapsto\left\langle y^{\prime},|x|\right\rangle$ is a continuous lattice seminorm on $E$ with kernel $\operatorname{ker} p=N\left(y^{\prime}\right):=\left\{x \in E:\left\langle y^{\prime},|x|\right\rangle=0\right\}$. Then $p$ induces a lattice norm on $E /$ ker $p$. Let $\left(E, y^{\prime}\right)$ be its (norm) completion, which is again a Banach lattice, and let $j_{y^{\prime}}: E \rightarrow\left(E, y^{\prime}\right)$ be the lattice homomorphism induced by the quotient map $q: E \rightarrow E / \operatorname{ker} p$. It turns out that $\left(E, y^{\prime}\right)$ is an $A L$-space, that is, on $\left(E, y^{\prime}\right)_{+}$the norm is additive. If $T \in \mathscr{L}(E)$ is a positive operator such that $T^{\prime} y^{\prime} \leq y^{\prime}$, then $T N\left(y^{\prime}\right) \subseteq N\left(y^{\prime}\right)$. Hence $T$ induces an operator $T /$ on $E / \operatorname{ker} p$ which is a positive 
contraction for the norm induced by $p$. Thus $T /$ has a unique contractive positive extension $\tilde{T} \in \mathscr{L}\left(\left(E, y^{\prime}\right)\right)$. We call $\tilde{T}$ the operator on $\left(E, y^{\prime}\right)$ induced by $T$.

Now we can state the following inheritance result for the point spectrum.

THEOREM 2.2. Let $E$ be a Banach lattice and let $S, T \in \mathscr{L}(E)$ such that $0 \leq S \leq T$ and $\alpha T$ is Abel ergodic for all $\alpha \in \Gamma$. Then $P \sigma(S) \cap r(T) \Gamma \subseteq P \sigma(T) \cap r(T) \Gamma$.

ProOF. Firstly let $r(T)=0$. Clearly, $T$ satisfies $(G)$, and hence $\sup _{\lambda>0}\|\lambda R(\lambda, T)\|$ $<\infty$. From $|\lambda R(\lambda, T) x| \leq|\lambda| R(|\lambda|, T)|x|, \lambda \in \mathbb{C} \backslash\{0\}, x \in E$, we obtain $\sup _{\lambda \in \mathbb{C} \backslash\{0\}}\|\lambda R(\lambda, T)\|<\infty$. Then $\lambda \mapsto \lambda R(\lambda, T)=I+T R(\lambda, T)$ has a removable singularity at 0 . Thus $T R(\lambda, T)$ has a holomorphic extension to the whole complex plane. Since $\lim _{|\lambda| \rightarrow \infty} T R(\lambda, T)=0$, Liouville's theorem implies $T R(., T)=0$, and hence $T=0$.

Now let $r(T)>0$. Without loss of generality we may assume $r(T)=1$. Let $\alpha \in P \sigma(S) \cap \Gamma$ and choose $0 \neq x \in E$ such that $S x=\alpha x$. Then $|x| \leq S|x| \leq T|x|$. Since $T$ is Abel ergodic, $y:=\lim _{\lambda \downarrow 1}(\lambda-1) R(\lambda, T)|x|$ exists and $0 \leq|x| \leq y=T y$. Let $x^{\prime} \in E_{+}^{\prime}$ be such that $\left\langle x^{\prime},|x|\right\rangle>0$. Again by Abel ergodicity, $y^{\prime}:=\sigma\left(E^{\prime}, E\right)$ $\lim _{\lambda \downarrow 1}(\lambda-1) R(\lambda, T)^{\prime} x^{\prime}$ exists and $0 \leq S^{\prime} y^{\prime} \leq T^{\prime} y^{\prime}=y^{\prime}$. Moreover, $\left\langle y^{\prime},|x|\right\rangle=$ $\lim _{\lambda \downarrow 1}\left\langle(\lambda-1) R(\lambda, T)^{\prime} x^{\prime},|x|\right\rangle=\left\langle x^{\prime}, y\right\rangle \geq\left\langle x^{\prime},|x|\right\rangle>0$. Let $\tilde{S}$ and $\tilde{T}$ be the operators on the $A L$-space $\left(E, y^{\prime}\right)$ induced by $S$ and $T$, respectively. Then $0 \leq \tilde{S} \leq \tilde{T}$ and $\tilde{S}$ and $\tilde{T}$ are contractions. Let $\tilde{x}:=j_{y^{\prime}} x$. We have $\tilde{S} \tilde{x}=\alpha \tilde{x}$ and $\|\tilde{x}\|=\left\langle y^{\prime},|x|\right\rangle>0$, hence $\alpha \in \operatorname{Po}(\tilde{S})$. Moreover $|\tilde{x}| \leq \tilde{S}|\tilde{x}| \leq \tilde{T}|\tilde{x}|$. Since the norm of $\left(E, y^{\prime}\right)$ is strictly monotone on $\left(E, y^{\prime}\right)_{+}$and $\tilde{T}$ is contractive we obtain $\tilde{T}|\tilde{x}|=|\tilde{x}|$. Then Lemma 1.3 implies $\tilde{T} \tilde{x}=\alpha \tilde{x}$. Now $\alpha^{-1} T$ is Abel ergodic. Then $z:=\lim _{\lambda \downarrow l}(\lambda-1) R\left(\lambda, \alpha^{-1} T\right) x$ exists in $E$ and $T z=\alpha z$. Thus

$$
\begin{aligned}
j_{y^{\prime}} z & =\lim _{\lambda \downarrow 1} j_{y^{\prime}}(\lambda-1) R\left(\lambda, \alpha^{-1} T\right) x=\lim _{\lambda \downarrow 1}(\lambda-1) R\left(\lambda, \alpha^{-1} \tilde{T}\right) j_{y^{\prime}} x \\
& =\lim _{\lambda \downarrow 1}(\lambda-1) R\left(\lambda, \alpha^{-1} \tilde{T}\right) \tilde{x}=\tilde{x} \neq 0 .
\end{aligned}
$$

Hence $z \neq 0$ which shows $\alpha \in P \sigma(T) \cap \Gamma$.

REMARK. The proof shows that a positive operator $T$ is the zero operator if $r(T)=$ 0 and $T$ satisfies $(G)$.

If the powers of $T$ converge strongly, then $\alpha T$ is Abel ergodic for all $\alpha \in \Gamma$ and $P \sigma(T) \cap \Gamma \subseteq\{1\}$. Thus we obtain the following result.

COROLLARY 2.3. Let $E$ be a Banach lattice and let $S, T \in \mathscr{L}(E)$ be such that $0 \leq S \leq T$ and $\left(T^{n}\right)$ is strongly convergent. Then $\operatorname{P\sigma }(S) \cap \Gamma \subseteq P \sigma(T) \cap \Gamma \subseteq\{1\}$. 
If $E$ is a Banach lattice with order continuous norm we can relax the conditions on the operator $T$. Recall that a Banach lattice $E$ has order continuous norm if every decreasing net $\left(x_{\alpha}\right)_{\alpha \in A}$ in $E_{+}$, such that $\inf _{\alpha} x_{\alpha}=0$ satisfies $\lim _{\alpha}\left\|x_{\alpha}\right\|=0$. Examples of such spaces are $c_{0}, L^{p}$ for $1 \leq p<\infty$, and all reflexive Banach lattices. Order continuity of the norm is equivalent to the fact that for every relatively weakly compact

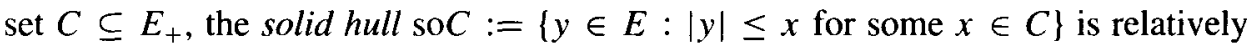
weakly compact as well (see $[2,13.8]$ ), or that every closed ideal in $E$ is a projection band (see [26, II.5.14]).

COROLLARY 2.4. Let $E$ be a Banach lattice with order continuous norm and let $S, T \in \mathscr{L}(E)$ such that $0 \leq S \leq T$ and $T$ is Abel ergodic. Then $P \sigma(S) \cap r(T) \Gamma \subseteq$ $P \sigma(T) \cap r(T) \Gamma$.

ProOF. We have to consider only the case $r(T)>0$ (see the remark after Theorem 2.2) and without loss of generality we may assume $r(T)=1$. Now let $\alpha \in \Gamma$. If $\lambda>1$ and $x \in E$ then

$$
\begin{aligned}
\left|(\lambda-1) R\left(\lambda, \alpha^{-1} T\right) x\right| & \leq(\lambda-1) \sum_{n \geq 0} \lambda^{-(n+1)}\left|\alpha^{-n} T^{n}\right||x| \\
& \leq(\lambda-1) R(\lambda, T)|x| .
\end{aligned}
$$

Since $T$ is Abel ergodic, $T$ and hence $\alpha^{-1} T$ satisfies $(G)$. Moreover $C:=\{(\lambda-1)$ $R(\lambda, T)|x|: 1<\lambda \leq 2\}$ is relatively compact and $D:=\left\{(\lambda-1) R\left(\lambda, \alpha^{-1} T\right) x\right.$ : $1<\lambda \leq 2\}$ is contained in the solid hull of $C$. The order continuity of the norm then implies that $D$ is relatively weakly compact. Thus $\alpha^{-1} T$ is Abel ergodic by Proposition 2.1. The assertion follows now from Theorem 2.2.

The next lemma is a pointwise version of Corollary 2.4 .

LEMMA 2.5. Let $E$ be a Banach lattice with order continuous norm and let $S, T \in$ $\mathscr{L}(E)$ be such that $0 \leq S \leq T$ and $T$ satisfies $(G)$. Let $\alpha \in r(T) \Gamma$ and $0 \neq x \in E$ be such that $S x=\alpha x$. If $\lim _{\lambda \downarrow r(T)}(\lambda-r(T)) R(\lambda, T)|x|$ exists in $E$, then $\alpha \in P \sigma(T)$.

Proof. If $r(T)=0$, then by the remark after Theorem 2.2 we have $T=0$, and hence $0 \in P \sigma(T)$. Now let $r(T)>0$. Without loss of generality we may assume $r(T)=1$. Then $|x| \leq S|x| \leq T|x|$. Since $T$ satisfies $(G)$ there exists $x^{\prime} \in E_{+}^{\prime}$ such that $T^{\prime} x^{\prime}=x^{\prime}$ and $\left\langle x^{\prime},|x|\right\rangle>0$ (see [26, V.4.8]). Let $N\left(x^{\prime}\right):=$ $\left\{y \in E:\left\langle x^{\prime},|y|\right\rangle=0\right\}$. Since $E$ has order continuous norm the closed ideal $N\left(x^{\prime}\right)$ is a projection band (see [26, II.5.14]), and hence $E=N\left(x^{\prime}\right) \oplus N\left(x^{\prime}\right)^{\perp}$, where $N\left(x^{\prime}\right)^{\perp}:=\left\{u \in E: \inf (|u|,|v|)=0\right.$ for all $\left.v \in N\left(x^{\prime}\right)\right\}$. Let $Q$ be the band projection from $E$ onto $N\left(x^{\prime}\right)^{\perp}$. Since $0 \leq S^{\prime} x^{\prime} \leq T^{\prime} x^{\prime}=x^{\prime}$ the operators $S$ and $T$ leave $N\left(x^{\prime}\right)$ invariant, that is, $S(I-Q) E \subseteq(I-Q) E$ and $T(I-Q) E \subseteq(I-Q) E$. If we represent 
$S$ and $T$ as operator matrices according to the decomposition $E=N\left(x^{\prime}\right) \oplus N\left(x^{\prime}\right)^{\perp}$, we obtain

$$
S=\left(\begin{array}{cc}
S_{1} & S_{2} \\
0 & S_{3}
\end{array}\right) \quad \text { and } \quad T=\left(\begin{array}{cc}
T_{1} & T_{2} \\
0 & T_{3}
\end{array}\right)
$$

where $S_{3}=Q S_{\mid Q E}$ and $T_{3}=Q T_{\mid Q E}$. In particular $0 \leq S_{3} \leq T_{3}$. Let $\tilde{x}^{\prime}:=x_{\mid Q E}^{\prime}$. Then $\tilde{x}^{\prime} \in(Q E)^{\prime}$ is strictly positive and for $y \in Q E$ we have

$$
\begin{aligned}
\left\langle\tilde{x}^{\prime}, T_{3} y\right\rangle & =\left\langle x^{\prime}, Q T y\right\rangle=\left\langle x^{\prime}, Q T y\right\rangle+\left\langle x^{\prime},(I-Q) T y\right\rangle \\
& =\left\langle x^{\prime}, T y\right\rangle=\left\langle x^{\prime}, y\right\rangle=\left\langle\tilde{x}^{\prime}, y\right\rangle ;
\end{aligned}
$$

that is, $T_{3}^{\prime} \tilde{x}^{\prime}=\tilde{x}^{\prime}$. Let $\tilde{x}:=Q x$. From $|x|=|Q x|+|(I-Q) x|$ it follows that

$$
\left\langle\tilde{x}^{\prime},|\tilde{x}|\right\rangle=\left\langle x^{\prime},|Q x|+|(I-Q) x|\right\rangle=\left\langle x^{\prime},|x|\right\rangle>0,
$$

and hence $\tilde{x} \neq 0$. The $S$-invariance of $N\left(x^{\prime}\right)$ yields $S_{3} Q x=Q S x$, and hence $S_{3} \tilde{x}=\alpha \tilde{x}$. The remark after Lemma 1.3 then implies $T_{3} \tilde{x}=\alpha \tilde{x}$.

Let now $\lambda>1$ and $y \in E$. Then

$$
\left|R\left(\lambda, \alpha^{-1} T\right) y\right|=\left|\sum_{n \geq 0} \frac{T^{n} y}{\alpha^{n} \lambda^{n+1}}\right| \leq \sum_{n \geq 0} \frac{T^{n}|y|}{\lambda^{n+1}}=R(\lambda, T)|y| .
$$

Thus

$$
\begin{gathered}
\sup _{\lambda>1}\left\|(\lambda-1) R\left(\lambda, \alpha^{-1} T\right)\right\| \leq \sup _{\lambda>1}\|(\lambda-1) R(\lambda, T)\|<\infty \text { and } \\
\left\{(\lambda-1) R\left(\lambda, \alpha^{-1} T\right) x: 1<\lambda \leq 2\right\} \subseteq \operatorname{so}\{(\lambda-1) R(\lambda, T)|x|: 1<\lambda \leq 2\} .
\end{gathered}
$$

Since the limit $\lim _{\lambda \downarrow 1}(\lambda-1) R(\lambda, T)|x|$ exists and $E$ has order continuous norm, $\left\{(\lambda-1) R\left(\lambda, \alpha^{-1} T\right) x: 1<\lambda \leq 2\right\}$ is relatively weakly compact. Proposition 2.1 then implies that $z:=\lim _{\lambda \downarrow 1}(\lambda-1) R\left(\lambda, \alpha^{-1} T\right) x$ exists in $E$, and $T z=\alpha z$.

It remains to show $z \neq 0$. The $T$-invariance of $N\left(x^{\prime}\right)$ implies $T_{3} Q y=Q T y$ for every $y \in E$. Hence $T_{3}^{n} Q y=Q T^{n} y$ for all $n \in \mathbb{N}$ and $y \in E$. Thus

$$
\begin{aligned}
Q z & =\lim _{\lambda \downarrow 1}(\lambda-1) \sum_{n \geq 0} \frac{Q\left(\alpha^{-1} T\right)^{n} x}{\lambda^{n+1}}=\lim _{\lambda \downarrow 1}(\lambda-1) \sum_{n \geq 0} \frac{\alpha^{-n} T_{3}^{n} \tilde{x}}{\lambda^{n+1}} \\
& =\lim _{\lambda \downarrow 1}(\lambda-1) \sum_{n \geq 0} \frac{\tilde{x}}{\lambda^{n+1}}=\tilde{x} \neq 0,
\end{aligned}
$$

and hence $z \neq 0$. 
If in Theorem 2.2 the Banach lattice $E$ is a $K B$-space we can further relax the conditions on $T$. Recall that a Banach lattice $E$ is a $K B$-space if $E$ is a (projection) band in its bidual $E^{\prime \prime}$. In this case every increasing uniformly bounded sequence $\left(x_{n}\right)$ in $E_{+}$converges in norm (see [26, II.5.15]). Note that every $K B$-space has order continuous norm. Examples of $K B$-spaces are $L^{p}$ for $1 \leq p<\infty$, and all reflexive Banach lattices.

THEOREM 2.6. Let $E$ be a $K B$-space and let $S, T \in \mathscr{L}(E)$ be such that $0 \leq S \leq T$ and $T$ satisfies $(G)$. Then $P \sigma(S) \cap r(T) \Gamma \subseteq P \sigma(T) \cap r(T) \Gamma$.

PROOF. If $r(T)=0$ the assertion follows from the remark after Theorem 2.2. As above, the case $r(T)>0$ can be reduced to the case $r(T)=1$. Let $S x=\alpha x$ for $\alpha \in \Gamma$ and $0 \neq x \in E$. Then $|x| \leq S|x| \leq T|x|$. Hence the sequence $\left(T^{n}|x|\right)$ is increasing. On the other hand, if $\lambda>1$, then $(\lambda-1) R(\lambda, T)|x| \geq(\lambda-1) \sum_{m \geq n} T^{m}|x| / \lambda^{m+1} \geq$ $\lambda^{-n} T^{n}|x|$ for every $n \in \mathbb{N}$. Property $(G)$ implies that $\left(T^{n}|x|\right)$ is uniformly bounded. Since $E$ is a $K B$-space $y:=\lim _{n} T^{n}|x|$ exists in $E$ and $|x| \leq y=T y$. Thus if $\lambda>1$ then $0 \leq(\lambda-1) R(\lambda, T)|x| \leq y$. Hence $\{(\lambda-1) R(\lambda, T)|x|: \lambda>1\}$ is contained in the order interval $[0, y]$ which is weakly compact (see [26, II.5.10]). By Proposition 2.1 the limit $\lim _{\lambda \downarrow 1}(\lambda-1) R(\lambda, T)|x|$ exists. Now Lemma 2.5 implies $\alpha \in P \sigma(T)$ and the proof is finished.

The following example shows that in Theorem 2.2 and Corollary 2.4 the condition on $T$ (Abel ergodicity) and in Theorem 2.6 the condition on $E$ ( $K B$-space) cannot be omitted.

EXAMPLE 2.7. Let $E=c_{0}$ be the space of all sequences converging to 0. Define operators $S$ and $T$ on $E$ by $S x:=\left(\xi_{1}, 0, \xi_{2}, \xi_{3}, \ldots\right)$ and $T x:=\left(\xi_{1}, \xi_{1}, \xi_{2}, \xi_{3}, \ldots\right), x=$ $\left(\xi_{n}\right) \in E$. Then $0 \leq S \leq T,\|T\|=1$ and $S e_{1}=e_{1}$ where $e_{1}=(1,0,0, \ldots)$. In particular $1 \in P \sigma(S)$. On the other hand, let $x=\left(\xi_{n}\right) \in E$ be such that $T x=x$. Then $\xi_{1}=\xi_{2}=\ldots$. However, the only constant sequence belonging to $E$ is the zero sequence, hence $1 \notin P \sigma(T)$. Since $(\lambda-1) R(\lambda, T) e_{1}$ does not converge as $\lambda \downarrow 1$ the operator $T$ is not Abel ergodic. Finally, if $\alpha \in \Gamma \backslash\{1\}$, then an easy computation shows that 1 is not an eigenvalue of $\alpha T^{\prime}$. Thus, by [16, Theorem 2.1.4, Theorem 2.1.5], the operator $\alpha T$ is Abel ergodic for each $\alpha \in \Gamma \backslash\{1\}$.

From the results on the point spectrum we can deduce inheritance properties for the residual spectrum. In fact, if $T \in \mathscr{L}(E)$ is an operator on a Banach space $E$, consider $R \sigma(T):=\{\lambda \in \mathbb{C}:(\lambda-T) E$ is not dense in $E\}$, the residual spectrum of $T$. Then $R \sigma(T)=P \sigma\left(T^{\prime}\right)$ by the Hahn-Banach Theorem. Thus Theorem 2.6 leads to the following result. 
THEOREM 2.8. Let $E$ be a Banach lattice such that $E^{\prime}$ has order continuous norm and let $S, T \in \mathscr{L}(E)$ be such that $0 \leq S \leq T$, and $T$ satisfies $(G)$. Then $R \sigma(S) \cap$ $r(T) \Gamma \subseteq R \sigma(T) \cap r(T) \Gamma$.

ProOF. The assumptions on $S$ and $T$ imply that $0 \leq S^{\prime} \leq T^{\prime}$ and $T^{\prime}$ satisfies $(G)$. If $E^{\prime}$ has order continuous norm, then $E^{\prime}$ is already a $K B$-space (see $[19,2.4 .14]$ ). Thus Theorem 2.6 yields

$$
R \sigma(S) \cap r(T) \Gamma=P \sigma\left(S^{\prime}\right) \cap r(T) \Gamma \subseteq P \sigma\left(T^{\prime}\right) \cap r(T) \Gamma=R \sigma(T) \cap r(T) \Gamma .
$$

REMARK. One also obtains analogues of Theorem 2.2 and Corollaries 2.3 and 2.4 for the residual spectrum.

\section{The essential spectrum of dominated operators}

If $0 \leq S \leq T$ are operators on a Banach lattice $E$ and $r(T)$ is a Riesz point of $T$, then by a result of Caselles [5, Theorem 4.1] either $r(S)<r(T)$ or $r(T)$ is a Riesz point of $S$. In this section we show that an analogous conclusion holds for every operator $S$ which is dominated by $T$, that is, such that $|S x| \leq T|x|$ for all $x \in E$.

Let us make this more precise. For an operator $T \in \mathscr{L}(E)$ on a Banach space $E$ let $\Phi(T):=\{\lambda \in \mathbb{C}: \operatorname{ker}(\lambda-T)$ and $E /(\lambda-T) E$ are finite dimensional $\}$ be the Fredholm domain, $\sigma_{\mathrm{ess}}(T):=\mathbb{C} \backslash \Phi(T)$ the (Wolf) essential spectrum, and $r_{\mathrm{ess}}(T):=\sup \left\{|\lambda|: \lambda \in \sigma_{\mathrm{ess}}(T)\right\}$ the essential spectral radius of $T$. If $\sigma_{\mathrm{ess}}(T)=\emptyset$, we set $r_{\text {ess }}(T)=-\infty$. It is well-known that $\sigma_{\text {ess }}(T) \subseteq \sigma(T)$ is compact and $\sigma_{\text {ess }}(T) \neq \emptyset$ if $E$ is infinite dimensional (see [11, XI, p.205]). Recall that $\lambda \in \sigma(T)$ is a Riesz point of $T$ if $\lambda$ is a pole of the resolvent map with residuum of finite rank. It turns out that $\left\{\lambda \in \sigma(T):|\lambda|>r_{\text {ess }}(T)\right\}$ contains only Riesz points (see [11, XI.8.4]). Conversely, every Riesz point of $T$ belongs to $\sigma(T) \backslash \sigma_{\text {ess }}(T)$ (see [11, XI.5.3]). If $r(T)=0$ and 0 is a Riesz point, then $T$ is nilpotent and hence $E$ is finite dimensional (notice that the Neumann series is the Laurent expansion of the resolvent $R(., T)$ ).

If $T$ is a positive operator on a Banach lattice $E$ and $r(T)$ is a Riesz point of $T$, then by a result of Niiro and Sawashima all elements of $\sigma(T) \cap r(T) \Gamma$ are poles of the resolvent. An inspection of the proof given by Lotz and Schaefer (see [26, V.5.5]) even shows that $\sigma(T) \cap r(T) \Gamma$ consists entirely of Riesz points (see also [18, Corollary 2.3]). Now the result of Caselles [5, Theorem 4.1] reads as follows (see also [18, Proposition 2.5]):

PROPOSITION. If $0 \leq S \leq T$ are operators on a Banach lattice $E$ such that $r(T)$ is a Riesz point of $T$, then $r_{\mathrm{ess}}(S)<r(T)$. 
Our aim is to prove the following generalization of Caselles' result.

THEOREM 3.1. Let $E$ be a Banach lattice and let $S, T \in \mathscr{L}(E)$ be operators such that $S$ is dominated by $T$, and $r(T)$ is a Riesz point of $T$. Then $r_{\text {ess }}(S)<r(T)$. In particular, $\sigma(S) \cap r(T) \Gamma$ contains only Riesz points.

The proof of Theorem 3.1 is divided into several 'auxiliary results'. Our first lemma is due to Greiner [12, Proposition 1.32] (see also [6, Lemma 8.9]) and has its origin in a result of Schaefer [26, V.5.1, V.7.4]. If $E$ is a Banach lattice and $z \in E_{+}$, then $E_{z}$ denotes the ideal generated by $z$ endowed with the norm $p_{z}(x):=\inf \{r>0$ : $|x| \leq r z\}$. The space $E_{z}$ is a Banach lattice (see [26, II.7.2]). Moreover there is an isometric lattice isomorphism from $E_{z}$ onto a space $C(K), K$ compact, which maps $z$ to $1_{K}$ (see [26, II.7.2, II.7.4]).

LEMMA 3.2. Let $S, T \in \mathscr{L}(E)$ be operators on a Banach lattice $E$ such that $S$ is dominated by $T$. Suppose there is $\alpha \in \Gamma$ and $0 \neq z \in E$ such that $S z=\alpha z$ and $T|z|=|z|$. Then there is a surjective isometry $V \in \mathscr{L}\left(\overline{E_{|z|}}\right)$ such that $S x=$ $\alpha V T V^{-1} x$ for all $x \in \overline{E_{|z|}}$.

If $T$ is an operator on a Banach space $E, G \subseteq E$ a closed $T$ - invariant subspace and $\lambda \in \sigma(T) \cap r(T) \Gamma$ a Riesz point of $T$, then Lemma 1.1 implies that $\lambda$ is a Riesz point or belongs to the resolvent set of the induced operators $T_{1}$ and $T_{/}$on $G$ and $E / G$, respectively. In case $G$ is an ideal in a Banach lattice $E$, Caselles [5, Lemma 4.4] has shown that the converse is true. We formulate his result in a slightly different form.

LEMma 3.3. Let $E$ be a Banach lattice, $T \in \mathscr{L}(E), I \subseteq E$ a closed $T$-invariant ideal, and $T_{1}$ and $T_{/}$the induced operators on $I$ and $E / I$, respectively. Suppose that $\lambda \in \mathbb{C}$ is a Riesz point of $T_{1}$ and $T_{1}$, or a Riesz point of either $T_{1}$ or $T_{1}$ and belongs to the resolvent set of the other operator. Then $\lambda$ is a Riesz point of $T$.

Now we prove a special case of Theorem 3.1. Recall that a positive operator $T$ on a Banach lattice $E$ is irreducible if $\{0\}$ and $E$ are the only closed $T$-invariant ideals in $E$. We call $u \in E_{+}$a topological order unit if the ideal generated by $u$ is dense in $E$. For $z^{\prime} \in E^{\prime}$ and $z \in E$ we denote by $z^{\prime} \otimes z$ the operator given by $\left(z^{\prime} \otimes z\right) x:=\left\langle z^{\prime}, x\right\rangle z, x \in E$.

LEMMA 3.4. Let $E$ be a Banach lattice and let $S, T \in \mathscr{L}(E)$ be operators such that $S$ is dominated by $T, r(T)$ is a Riesz point of $T$, and $T$ is irreducible. Then $r_{e s s}(S)<r(T)$.

PROOF. If $E$ is finite dimensional there is nothing to prove. Otherwise we have $r(T)>0$ and without loss of generality we may assume $r(T)=1$. If $r(S)<1$ 
the assertion holds. Now let $r(S)=1$ and $\lambda \in \sigma(S) \cap \Gamma$. Consider an ultrapower $E_{\mathscr{U}}$ of $E$ and the operator $S_{\mathscr{U}}$ induced by $S$. Then $\lambda \in P \sigma\left(S_{\mathscr{U}}\right)$ by Lemma 1.2. Choose $0 \neq \hat{v} \in E_{\mathscr{U}}$ such that $S_{\mathscr{U}} \hat{v}=\alpha \hat{v}$. Clearly, $S_{\mathscr{U}}$ is dominated by $T_{\mathscr{U}}$. The irreducibility of $T$ implies that 1 is a pole of order 1 of $R(., T)$ and the residuum $P$ at 1 is given by $P=z^{\prime} \otimes u$, where $z^{\prime} \in E_{+}^{\prime}$ is strictly positive and $u \in E_{+}$is a topological order unit (see [26, V.5.1, V.5.2]). Lemma 1.2 implies that 1 is a pole of order 1 of $R\left(., T_{\mathscr{U}}\right)$ with residuum $P_{\mathscr{U}}=\hat{z}^{\prime} \otimes \hat{u}$, where $\hat{u}:=(u, u, \ldots)^{\hat{u}} \in E_{\mathscr{U}}$ and $\hat{z}^{\prime} \in\left(E_{\mathscr{U}}\right)_{+}^{\prime}$ is given by $\left\langle\hat{z}^{\prime}, \hat{x}\right\rangle:=\lim _{\mathscr{U}}\left\langle z^{\prime}, x_{n}\right\rangle, \hat{x}=\widehat{\left(x_{n}\right)} \in E_{\mathscr{U}}$. Thus $P_{\mathscr{U}}$ has rank 1 , and hence 1 is a Riesz point of $T_{\mathscr{U}}$.

Since $T_{\mathscr{U}}^{\prime} \hat{z}^{\prime}=\hat{z}^{\prime}$ the closed ideal $I:=\left\{\hat{x} \in E_{\mathscr{U}}:\left\langle\hat{z}^{\prime},|\hat{x}|\right\rangle=0\right\} \subseteq E_{\mathscr{U}}$ is invariant for $T_{\mathscr{U}}$ and $S_{\mathscr{U}}$, respectively. Let $\left(T_{\mathscr{U}}\right)_{\mid}$and $\left(S_{\mathscr{U}}\right)$ be the induced operators on $I$. From $P_{\mathscr{U}} I=\{0\}$ and Lemma 1.1 it follows that $1 \in \rho\left(\left(T_{\mathscr{U}}\right)_{\mid}\right)$. Since $\left(T_{\mathscr{U}}\right)$ is positive this implies $r\left(\left(T_{\mathscr{U}}\right)_{\mid}\right)<1$ (see [26, V.4.1]), and hence $r\left(\left(S_{\mathscr{U}}\right)_{\mid}\right)<1$. In particular $\hat{v} \notin I$.

Let $W:=\left(T_{\mathscr{U}}\right)$, and $U:=\left(S_{\mathscr{U}}\right)$, be the induced operators on $F:=E_{\mathscr{U}} / I$ and let $z$ and $y$ be the canonical images of $\hat{v}$ and $\hat{u}$ in $F$, respectively. Then $U$ is dominated by $W, 1$ is a Riesz point of $W$ with corresponding residuum $Q:=\left(P_{\mathscr{U}}\right)_{/}=\hat{z}^{\prime} \otimes y$, and $0 \neq \alpha z=U z$. Since $|\hat{v}|=\left|S_{\mathscr{U}} \hat{v}\right| \leq T_{\mathscr{U}}|\hat{v}|$ and $T_{\mathscr{U}}^{\prime} \hat{z}^{\prime}=\hat{z}^{\prime}$ we obtain $T_{\mathscr{U}}|\hat{v}|-|\hat{v}| \in I$, and hence $W|z|=|z|=\lambda y$ for some $\lambda>0$.

Let $J$ be the closed ideal in $F$ generated by $y$. Then $J$ is invariant for $W, Q$ and $U$. Let $W_{1}, U_{1}$ and $W_{/}, Q_{/}, U_{/}$be the induced operators on $J$ and $F / J$, respectively. Then $Q_{l}=0$, and hence $1 \in \rho\left(W_{/}\right)$. Since $W_{/}$is positive and $U_{/}$is dominated by $W_{\text {/ we obtain }} r\left(U_{l}\right) \leq r\left(W_{l}\right)<1$. On the other hand, 1 is a Riesz point of $W_{1}$ and $W_{\mid}, U_{\mid}, \alpha$ and $z$ satisfy the assumptions of Lemma 3.2. Thus the operators $U_{\mid}$and $\alpha W_{\mid}$ are similar, and hence $\alpha$ is a Riesz point of $U_{\mid}$. Now Lemma 3.3 and $r\left(U_{/}\right)<1 \mathrm{imply}$ that $\alpha$ is a Riesz point of $U=\left(S_{\mathscr{U}}\right)_{/}$. Since $r\left(\left(S_{\mathscr{U}}\right)_{\mid}\right)<1$ by the same argument, we obtain that $\alpha$ is a Riesz point of $S_{\mathscr{U}}$. Thus $\alpha$ is a Riesz point of $S=S_{\mathscr{U} \mid E}$.

Now we prove Theorem 3.1. We follow the lines of a proof of Lotz and Schaefer (see [26, V.5.5] and [5, Theorem 4.1]).

ProOF OF THEOREM 3.1. If $E$ is finite dimensional the assertion is obvious. Now let $E$ be infinite dimensional. Since $r(T)$ is a Riesz point we have $r(T)>0$. Then without loss of generality we may assume $r(T)=1$. The proof is now divided into three steps.

(1) We first assume that $r(T)=1$ is a pole of order one of $R(., T)$ and its residuum $P$ is strictly positive, that is, $P x \in E_{+} \backslash\{0\}$ for all $x \in E_{+} \backslash\{0\}$. Then $P E=\operatorname{Fix}(T)$ (see [8, Theorem 2.17]) and from [26, III.11.5] it follows that $P E$ is a finite dimensional sublattice of $E$. Thus $P E$ is the linear span of normalized, mutually orthogonal vectors $e_{1}, \ldots, e_{n} \in(P E)_{+}$. Let $J_{k}, 1 \leq k \leq n$, be the closed ideal in $E$ generated by $e_{k}$. Then $T J_{k} \subseteq J_{k}$ and by [26, III.8.5] each $T_{k}:=T_{\mid J_{k}}$ is irreducible. 
Since $S$ is dominated by $T$, each $J_{k}$ is invariant for $S$. Hence we can apply Lemma 3.4 to $T_{k}$ and $S_{k}:=S_{\mid J_{k}}$ and obtain $r_{\text {ess }}\left(S_{k}\right)<1$ for $1 \leq k \leq n$. Now $J:=\sum_{k=1}^{n} J_{k}$ is a closed ideal (see [26, III.1.2]) which is invariant for $T$ and $S$. Since $P E \subseteq J$ and $T$ is positive, the induced operator $T_{/}$on $E / J$ satisfies $r\left(T_{/}\right)<1$. Therefore the same holds for the induced operator $S_{/}$on $E / J$. On the other hand, $r_{\text {ess }}\left(S_{\mid J}\right)<1$ by the foregoing reasoning. Hence the assertion follows from Lemma 3.3.

(2) Next, let $r(T)=1$ be a pole of order one of $R(., T)$ with (not necessarily strictly positive) residuum $P$. Since $T P=P T$, the ideal $J:=\{x \in E: P|x|=0\}$ is invariant under $T$ and $r\left(T_{\mid J}\right)<1$. Thus $S J \subseteq J$ and $r\left(S_{\mid J}\right)<1$. For the induced operators $T_{/}$and $S_{/}$on $E / J$ we are in the situation of (1). Hence the assertion follows from Lemma 3.3.

(3) Finally, let $r(T)=1$ be a pole of order $k>1$. Then $Q:=\lim _{\lambda \downarrow 1}(\lambda-1)^{k} R(\lambda, T)$ is a positive operator on $E$ satisfying $Q^{2}=0$. Since $T Q=Q T$ the ideal $J:=\{x \in$ $E: Q|x|=0\}$ is $T$-invariant. For the induced operators $T_{1}$ and $T_{/}$on $J$ and $E / J$, respectively, we obtain that 1 is a pole of order $k-1$ of $R\left(., T_{\mid}\right)$and a pole of order 1 of $R\left(., T_{/}\right)$. Moreover, $S J \subseteq J$ and the induced operators $S_{\mid}$and $S_{/}$on $J$ and $E / J$ are dominated by $T_{\mid}$and $T_{/}$, respectively. Thus the assertion follows by induction over $k$ and applying Lemma 3.3 .

\section{Asymptotic properties of dominated operators}

In this section we apply the previous results to investigate inheritance of asymptotic properties. Recall that by the theorem of Katznelson-Tzafriri [15, Theorem 1] an operator $T$ on a Banach space $E$ with uniformly bounded powers $T^{n}$ satisfies $\lim _{n}\left\|T^{n}-T^{n+1}\right\|=0$ if and only if $\sigma(T) \cap \Gamma \subseteq\{1\}$. Now the following result is an immediate consequence of Theorem 1.4.

THEOREM 4.1. Let $E$ be a Banach lattice and let $S, T \in \mathscr{L}(E)$ be such that $0 \leq S \leq T, \sup _{n}\left\|T^{n}\right\|<\infty$ and $\lim _{n}\left\|T^{n}-T^{n+1}\right\|=0$. Then $\lim _{n}\left\|S^{n}-S^{n+1}\right\|=0$.

In [24] and [25] it is shown that for operators $0 \leq S \leq T$ on a Banach lattice $E$ with order continuous norm strong convergence of $\left(T^{n}\right)$ to a projection $P_{T}$ of finite rank implies strong convergence of $\left(S^{n}\right)$. We will see that the rank condition on $P_{T}$ can be replaced by a spectral condition on $T$.

At first we prove an inheritance result for a property which is slightly more general than strong convergence of the powers $T^{n}, n \in \mathbb{N}$. An operator $T$ on a Banach space $E$ is called almost periodic, if $\left\{T^{n} x: n \in \mathbb{N}\right\}$ is relatively compact for all $x \in E$. In this case the Jacobs-Glicksberg-deLeeuw splitting theorem (see $[16, \S 2.4]$ ) yields a decomposition $E=E_{0} \oplus E_{r}$ of $E$ where

$$
E_{0}=E_{0}(T)=\left\{x \in E: \lim _{n} T^{n} x=0\right\} \quad \text { and }
$$




$$
E_{r}=E_{r}(T)=\varlimsup \operatorname{lin}\{x \in E: T x=\alpha x \text { for some } \alpha \in \Gamma\} .
$$

Now we obtain the following inheritance result for almost periodicity (see [24, Proposition 3.10] and [25, Theorem 4.6]). Notice that we do not impose any restriction on the projection $Q_{T}$ from $E$ onto $E_{r}$.

THEOREM 4.2. Let $E$ be a Banach lattice with order continuous norm and let $S, T \in \mathscr{L}(E)$ be such that $0 \leq S \leq T$ and $T$ is almost periodic. If $\sigma(T) \cap \Gamma \neq \Gamma$, then $S$ is almost periodic.

PROOF. By the uniform boundedness principle, $\sup _{n}\left\|S^{n}\right\| \leq \sup _{n}\left\|T^{n}\right\|<\infty$, and hence $r(T) \leq 1$. If $r(T)<1$, then $r(S) \leq r(T)<1$, which implies $\lim _{n}\left\|S^{n}\right\|=0$. Thus we may assume $r(T)=1$. By a result of $\operatorname{Lotz}$ (see [26, V.4.9]) $\sigma(T) \cap \Gamma$ is cyclic, that is, $\lambda \in \sigma(T) \cap \Gamma$ implies $\lambda^{n} \in \sigma(T)$ for all $n \in \mathbb{Z}$. Since $\sigma(T) \cap \Gamma$ is not the whole unit circle it must be a finite union of finite subgroups of $\Gamma$. Hence there exists $m \in \mathbb{N}$ such that $\sigma\left(T^{m}\right) \cap \Gamma=\{1\}$. By Theorem 1.4 we have $\sigma\left(S^{m}\right) \cap \Gamma \subseteq \sigma\left(T^{m}\right) \cap \Gamma=\{1\}$. On the other hand, $\left\{T^{m n} x: n \in \mathbb{N}\right\}$ is relatively compact for $x \in E$. Since $E$ has order continuous norm $\left\{S^{m n} x: n \in \mathbb{N}\right\} \subseteq \operatorname{so}\left\{T^{m n}|x|: n \in \mathbb{N}\right\}$ is relatively weakly compact for all $x \in E$. If $\lambda>1$ then $(\lambda-1) R\left(\lambda, S^{m}\right) x$ is in the closed convex hull of $\left\{S^{m n} x: n \in \mathbb{N}\right\}$ which is again weakly compact by Eberlein's theorem. Then Proposition 2.1 implies that $S^{m}$ is Abel ergodic and $E=\operatorname{Fix}\left(S^{m}\right) \oplus \overline{\left(I-S^{m}\right) E}$. Since $\sigma\left(S^{m}\right) \cap \Gamma \subseteq\{1\}$, the Katznelson-Tzafriri theorem (see [15, Theorem 1]) yields $\lim _{n}\left\|S^{m n}-S^{m(n+1)}\right\|=0$. Thus $\lim _{n} S^{m n} x=0$ for all $x \in \overline{\left(I-S^{m}\right) E}$, and hence $\left(S^{m n}\right)_{n \in \mathbb{N}}$ is strongly convergent. Thus $\left\{S^{n} x: n \in \mathbb{N}\right\} \subseteq \bigcup_{k=1}^{m} S^{k}\left\{S^{m(n-1)} x: n \in \mathbb{N}\right\}$ is relatively compact for all $x \in E$.

If $\left(T^{n}\right)$ is strongly convergent we obtain strong convergence of $\left(S^{n}\right)$.

COROLLARY 4.3. Let $E$ be a Banach lattice with order continuous norm and let $S, T \in \mathscr{L}(E)$ be such that $0 \leq S \leq T$ and $\left(T^{n}\right)$ is strongly convergent. If $\sigma(T) \cap \Gamma \neq$ $\Gamma$, then $\left(S^{n}\right)$ is strongly convergent.

PROOF. By Theorem 4.1 the operator $S$ is almost periodic. Then the JacobsGlicksberg-deLeeuw decomposition yields $E=E_{0}(S) \oplus E_{r}(S)$. By Corollary 2.3 we have $P \sigma(S) \cap \Gamma \subseteq P \sigma(T) \cap \Gamma \subseteq\{1\}$. Thus $E_{r}(S)=\operatorname{Fix}(S)$. Hence $\left(S^{n}\right)$ is strongly convergent.

If $\left(T^{n}\right)$ is uniformly convergent, then $1 \in \rho(T)$ or 1 is isolated in $\sigma(T)$ (see [16, 2.2.7]). So we obtain the following result.

COROLLARY 4.4. Let $E$ be a Banach lattice with order continuous norm and let $S, T \in \mathscr{L}(E)$ be such that $0 \leq S \leq T$ and $\left(T^{n}\right)$ is uniformly convergent. Then $\left(S^{n}\right)$ is strongly convergent. 
REMARKS. (a) In Corollary 4.4 one cannot expect a better convergence of the sequence $\left(S^{n}\right)$ (see [23, 2.6 Remark (a)]).

(b) We do not know if the conclusion of Theorem 4.2 and Corollary 4.3 still holds without the spectral condition on $T$. At least in that case one knows that $\left\{S^{n} x: n \in \mathbb{N}\right\}$ is relatively weakly compact for all $x \in E$, that is, $S$ is weakly almost periodic.

We conclude with an application of Theorem 3.1. Recall that an operator $T$ on a Banach space $E$ is uniformly ergodic if the Cesaro means $T_{n}:=\sum_{k=0}^{n-1} T^{k} / n, n \in \mathbb{N}$, are uniformly convergent. The limit $P_{T}:=\lim _{n} T_{n}$ is called the ergodic projection corresponding to $T$. It is well known that $T$ is uniformly ergodic if and only if $\lim _{n}\left\|T^{n}\right\| / n=0$ and 1 is a pole of the resolvent $R(., T)$ (see [8, Theorem 3.16]). In this case $P_{T}$ coincides with the spectral projection corresponding to the spectral set \{1\} (see [8, Theorem 2.23]). Thus $T$ is uniformly ergodic with ergodic projection of finite rank if and only if $\lim _{n}\left\|T^{n}\right\| / n=0$ and 1 is a Riesz point of $T$.

Now we obtain the following generalization of a result of Caselles [5, Corollary 4.6].

THEOREM 4.5. Let $E$ be a Banach lattice and let $S, T \in \mathscr{L}(E)$ be operators such that $S$ is dominated by $T$. If $T$ is uniformly ergodic with ergodic projection of finite rank, then $S$ is uniformly ergodic with ergodic projection of finite rank.

Proof. Our assumptions imply $r(S) \leq r(T) \leq 1$. If $r(S)<1$ there is nothing to prove. If $r(S)=r(T)=1$, then 1 is a Riesz point of $T$. Theorem 3.1 yields $r_{\text {ess }}(S)<$ 1. In particular 1 is a Riesz point of $S$. On the other hand, $\left\|S^{n}\right\| \leq\left\|T^{n}\right\|, n \in \mathbb{N}$, and hence $\lim _{n}\left\|S^{n}\right\| / n=0$. Thus the assertion follows from the previous discussion.

FINAL REMARK. The authors obtained corresponding results for pseudo-resolvents. This is the subject of a forthcoming paper.

\section{References}

[1] C. D. Aliprantis and O. Burkinshaw, 'On weakly compact operators on Banach lattices', Proc. Amer. Math. Soc. 83 (1981), 573-578.

[2] - Positive operators (Academic Press, London, 1985).

[3] F. Andreu, V. Caselles, J. Martinez and J. M. Mazon, 'The essential spectrum of AM-compact operators', Indag. Math. (N.S.) 2 (1991), 149-158.

[4] A. V. Bukhvalov, 'Integral representations of linear operators', J. Soviet. Math. 8 (1978), 129-137.

[5] V. Caselles, 'On the peripheral spectrum of positive operators', Israel J. Math. 58 (1987), 144-160.

[6] Ph. Clément, H. J. A. M. Heijmans, S. Angenent, C. J. van Duijn and B. de Pagter, One-parameter semigroups (North-Holland, Amsterdam, 1987). 
[7] P. G. Dodds and D. H. Fremlin, 'Compact operators in Banach lattices', Israel J. Math. 34 (1979), 287-320.

[8] N. Dunford, 'Spectral theory. I Convergence to projections', Trans. Amer. Math. Soc 54 (1943), 185-217.

[9] W. F. Eberlein, 'Abstract ergodic theorems and weak almost periodic functions', Trans. Amer. Math. Soc. 67 (1949), 217-240.

[10] R. Emilion, 'Mean bounded operators and mean ergodic theorems', J. Funct. Anal. 61 (1985), $1-14$.

[11] I. Gohberg, S. Goldberg and M. A. Kaashoek, Classes of linear operators, 1 (Birkhäuser, Basel, 1990).

[12] G. Greiner, Über das Spektrum stark stetiger Halbgruppen positiver Operatoren (Dissertation, Tübingen, 1980).

[13] W. Haid, Sätze vom Radon-Nikodym-Typ für Operatoren auf Banachverbänden (Dissertation, Tübingen, 1982).

[14] N. J. Kalton and P. Saab, 'Ideal properties of regular operators between Banach lattices', Illinois J. Math. 29 (1985), 382-400.

[15] Y. Katznelson and L. Tzafriri, 'On power bounded operators', J. Funct. Anal. 68 (1986), 313-328.

[16] U. Krengel, Ergodic theorems (de Gruyter, Berlin, 1985).

[17] J. Martinez, 'The essential spectral radius of dominated positive operators', Proc. Amer. Math. Soc. 118 (1993), 419-426.

[18] J. Martinez and J. M. Mazon, 'Quasi-compactness of dominated positive operators and $C_{0}$-semigroups', Math. Z. 207 (1991), 109-120.

[19] P. Meyer-Nieberg, Banach lattices (Springer, Berlin, 1991).

[20] B. de Pagter, 'The components of a positive operator', Indag. Math. 86 (1983), 229-241.

[21] B. de Pagter and A. R. Schep, 'Measures of non-compactness of operators on Banach lattices', $J$. Funct. Anal. 78 (1988), 31-55.

[22] F. Räbiger, Absolutstetigkeit und Ordnungsabsolutstetigkeit von Operatoren, Sitzungsberichte der Heidelberger Akademie der Wissenschaften, Math.-Naturwiss. Klasse, Jahrgang 1991, 1. Abhandlung, 1-132, (Springer, Berlin, 1991).

[23] — 'Stability and ergodicity of dominated semigroups, I. The uniform case', Math. Z. 214 (1993), 43-54.

[24] — , 'Stability and ergodicity of dominated semigroups, II. The strong case', Math. Ann. 297 (1993), 103-116.

[25] _ 'Attractors and asymptotic periodicity of positive operators on Banach lattices', Forum Math. 7 (1995), 665-683.

[26] H. H. Schaefer, Banach lattices and positive operators (Springer, Berlin, 1974).

[27] A. R. Schep, Kernel operators (Ph.D. Thesis, University of Leiden, Netherlands, 1977).

[28] A. C. Zaanen, Riesz spaces II (North-Holland, Amsterdam, 1983).

Mathematisches Institut

Universität Tübingen

Auf der Morgenstelle 10

D-72076 Tübingen

Germany

frra@michelangelo.mathematik.uni-tuebingen.de

manfred.wolff@uni-tuebingen.de 